\title{
Application of Artificial Intelligence in Clothing Production
}

\author{
Yufang $\mathrm{Lu}$ \\ ${ }^{1}$ School of Automation, Nanjing University of Aeronautics and Astronautics, Nanjing, China,211100 \\ *Corresponding author. Email: 1084759434@qq.com
}

\begin{abstract}
Clothing, as one of the necessities of human life, was born at the beginning of human society. With the development of human society, people's demand for clothing has gradually become diverse. Whether it is personalized clothing stores scattered all over the streets and alleys, or high-end brand clothing in major shopping malls, from low-grade stalls with tens of yuan to imported clothing with thousands or even tens of thousands of yuan, as long as it conforms to the trend and consumers' taste, People are willing to pay for it. In this context, the garment industry also began to change and evolve. Since the beginning of the industrial revolution, the garment industry has always been the most typical laborintensive industry, but there have always been problems such as excessive labor cost and time cost in the garment industry. In order to solve these problems, we combine the existing clothing production technology with automation and artificial intelligence, in the hope of accelerating the full automation in the clothing industry. Aiming at approaching this target, through theoretical and relevant case analyses, this paper explores the optimization schemes of some links in the production chain in the clothing industry, and analyzes some examples of automatic clothing production using artificial intelligence technology such as machine vision. Eventually, the optimization schemes of cutting, sorting and sewing are discovered, as well as the advantages and disadvantages of these schemes.
\end{abstract}

Keywords: Robot, Artificial intelligence, Automation, Clothing industry, Machine vision, Automatic clothing production

\section{INTRODUCTION}

With the development and maturity of automation technology, the clothing industry begins to combine these technologies and substitute mechanization for manual labor in some links of production. For example, in the past, buttonhole sewing could only be done manually. However, it requires a high degree of concentration so that as laborers work longer, potential manual errors will increase. Fortunately, in recent years, major breakthroughs and great progress have been made in the buttonhole sewing robot technology, while the visual guidance system of the buttonhole sewing robot has been developed and continuously being improved [1], and the maturity of delta robot buttonhole sewing technology [2] provides a theoretical basis for realizing the automation of this process. However, there are still some processes, such as cutting and sorting, which require human resources investment, lacking enough researches. Once the processes can be automated, it can greatly increase the production efficiency of the assembly line and largely decrease the production cost, which will make an inestimable contribution to the development of enterprises and the boom of the urban economy.

\section{HISTORICAL REVIEW OF CLOTHING PRODUCTION AUTOMATION AND MAIN REQUIREMENTS FOR MODERNIZATION}

\subsection{Historical Development Of Clothing Production Automation}

Before the 18th century, clothes could be produced purely by hand. It might take a handicrafts man several days to finish an article of clothing. If you wanted to make some complex patterns or include some delicate crafts on the clothes, such as lace, it would take a month or even more. Due to the limited technology at that time, it was almost impossible for clothing to achieve mass production. The first major change in the clothing industry took place during the Industrial Revolution. In 1790, Britain invented the world's first single chain hand sewing machine for leather shoes' sewing, which drilled holes first and then thread and sew. After that, in the 19th 
century, sewing machines continued to develop, evolve and improve. In 1825, the first double thread chain stitch sewing machine came out. Compared with its prototype, although it is bulkier, it worked steadier and more efficiently, that the sewing speed could reach nearly ten times that of manual sewing. In 1889, the first motordriven sewing machine appeared, and it officially entered the semi-automatic era for clothing production. After entering the 20th century, with the continuous maturing of computer technology, the functions of sewing machines are becoming more and more diverse. The types of clothing equipment have also leaped from single cutting and sewing in the early stage to more specialized and diverse clothing production equipment, including pre-sewing, sewing, post-sewing equipment and others [3].

\subsection{Requirements And Problems Of Modern Clothing Automatic Production}

After the 21st century, the main problem the clothing industry faces is still how to further enhance production efficiency and reduce production costs. Based on its quality of high efficiency and low cost, artificial intelligence can solve both problems at the same time. In the clothing production industry, the human cost has always dominated the total production cost. By introducing artificial intelligence, we can replace intelligent robots with manual labor to complete some repeated and relatively cumbersome work. Furthermore, after a long period of time working, the possibility of mistakes increases and the efficiency decreases. Artificial intelligence helps skip these problems. Not only is the execution efficiency much higher than that of human labor, but it can even run 24 hours a day if conditions permit.

However, due to the immature technology of artificial intelligence, most clothing enterprises still need to use a lot of manpower to complete some links in the production chain, such as cutting and sorting, and transportation between various links. A few links can be executed by some automatic machines, such as employing sewing machines for cut pieces sewing, etc. Therefore, to reach the assembly line production of clothes, still depends on the combination of a large number of labor force and a portion of automatic machinery.

\section{APPLICATION OF ARTIFICIAL INTELLIGENCE IN CLOTHING PRODUCTION AUTOMATION SYSTEM}

\subsection{To Realize Cutting Automation By Using Industrial Robot Technology Of Machine Vision}

The traditional manual clipping is inefficient and might also impair accuracy. Based on the improvement of the machine vision theory, we can use industrial robot technology to realize the automation of cut pieces cutting and replace manual labor.

Through machine vision technology, the robots can accurately distinguish the size and shape of the cutting piece, and then cut it according to the scanning results. This process requires that the robots can accurately judge the contour of the cutting piece, extract the data and record them on the computer. At present, there are many algorithms for contour extraction through machine vision. The mainstream ones are edge detection based on image edge extraction, edge detection based on mathematical morphology and so on. Image edge extraction is to analyze and record the color, brightness and other features of the target image, and then mark and extract the location of the two points where the difference of some feature parameters between the measurement and the standard is too large. The first and second derivatives are usually employed to detect the effects of boundary discontinuities. Edge extraction can be realized by convolution with the help of differential operators. The commonly used operators include Sobel operator, Prewitt operator, Roberts operator, LOG operator and Canny detection operator. At present, in the textile industry, contour extraction is mainly used in the fabric printing process. After comparative researches, Canny algorithm is fitter for the extraction of fabric contour information[4]. However, this method has high requirements for the quality of the surrounding environment and the picture itself. If the surrounding environment is filled with all kinds of floating objects, or the color difference of the picture itself is very small, there will be a great impact on the final extraction results. Compared with the image edge extraction method, the mathematical morphology method has no high requirements for the environment. Mathematical morphology means that the image is regarded as a collection of a series of points, and then these points are calculated by morphological method to separate the cut pieces from the background, and finally extract them. Although there are no requirements for the environment, this method is not as accurate as the first one in the locating of the contour. Both methods have their own advantages and disadvantages, and enterprises can select either according to their own workshop conditions.

\subsection{Automatic Cutting Sorting System Based On Artificial Intelligence}

Before sewing cut pieces into garments, there is another step to be completed, that is, piece sorting. In this process, many pieces mixed together need to be classified, and the pieces required for a ready-made garment need to be divided together. Otherwise, the cutting pieces will be superfluous or missing during sewing. In fact, we can realize automatic sorting through artificial intelligence and Internet of Things(IOT) technology. When printing 
and dyeing cutting pieces, we can each add a QR code and cut it together with the pieces in the cutting step. The operators in the background can upload these clips in the form of data through the computer, and the system can automatically determine the belonging of the clips to classify them in advance. During sorting, the grabbing robots can scan the QR code on the piece to obtain the information, as well as the position it should be placed, and leave it with other pieces of the garments before transporting it to the location for the next step. Compared with manual sorting, this method greatly saves labor costs and time costs. At the same time, the QR code will also be hidden in the inner side of the clothes during sewing, which will not affect their appearance.

\subsection{Sewing Automation Based On Intelligent Robots And Sewing Machines}

Although today's sewing machines can simplify the sewing process a lot, the operators still need to control the cutting pieces to pass through the sewing machines smoothly and evenly. We can achieve full automation of this process through programmable intelligent robots. It only needs to upload the mobile cutting instruction to the robot in the form of programming and make it run successfully.

But in fact, simply replacing manual work for mechanical arms will cause many problems. First of all, the console and the manipulator platform must be kept horizontal, because the manipulator actually uses pressing to make the cloth follow it. Once the console and the manipulator cannot be kept horizontal, the cloth is likely to loosen as it moves. The second problem is it is difficult to keep the running speed of the sewing machine consistent with that of the manipulator. Take Kawasaki Robot as an example, when writing codes, programmers can only adjust the relationship between the running speed of the robot and the maximum speed via the "speed" or "acceleration" instruction. However, it is difficult to adjust the speed of the sewing machine during automatic operation. If the speed difference is too large, the sewing result will be much different from that of manual operation.

There are many ways to solve this problem. The simplest one is to devote a lot of time to debugging the speed of the robot by trial and error to perfect the final outcome. Another is to add an encoder to the sewing machine, whose number of turns after operation can be read by the encoder. The programmer can obtain the results of the encoder through the computer, so as to get the actual speed of the sewing machine, and then the data will be programmed into the robot program, so that both speeds can be synchronized.

\section{DEVELOPMENT PROSPECTS}

Intellectualization and automation are the trends of development of the world, and the clothing industry must also comply with this trend. In the future, in addition to the previously mentioned parts, many more processes are sure to be performed by intelligent machines. For one thing, we should predict the future industry prospect through machine learning, so that leaders can more wisely make future plans and goals for enterprises. The trend prediction applying artificial neural networks is obviously better than the existing modeling methods because the former can not only solve many complex problems, but also predict the fashion of clothing and become an ideal tool for textile and clothing fashion innovation. Besides, it can also improve the sensitivity to market popular changes when using artificial intelligence systems, to better analyze the industry sales, predict the trend, and further guide the actual production [5]. For another, machine vision technology can also be applied in the field of error detection. When cutting a piece, due to the interference of various factors, there might be problems in its size or shape. Through higher-level machine vision technology, we can find these problems and correct them in time.

\section{CONCLUSION}

To sum up, with the current technology, there is still room for optimization in the above steps of garment production automation, and there are still quite a few steps that can be optimized. But this paper also has some limitations. Firstly, this paper only points out some theoretical feasibility, and does not verify the practical feasibility of these automation steps by means of experiments. In addition, this paper does not give the optimization scheme for other links of garment production. For example, the connection of the above steps is also one of the problems worth studying in garment production automation. Due to the limitations of many factors such as site, cutting and ready-made clothes can only be realized through manual transportation, which greatly affects the efficiency and cost. At the same time, based on the mature development of Internet technology, consumers' demand for customized clothes is also gradually increasing. Clothing design can also be independently designed and edited by consumers, so as to promote the development of personalized customized clothes. However, how to realize this operation needs more in-depth research. [6]. Anyway, I convince with the rapid development of science and technology, it is absolutely possible to realize full automation is not only the clothing industry but also other industries.

\section{ACKNOWLEDGMENT}

First of all, I would like to thank the tutor of the scientific research camp. Under your careful cultivation and guidance, my schoolwork and thesis were successfully completed. You not only give me academic guidance and help, but also give me a lot of support and 
understanding in life. Your tireless professionalism is my example and motivation in the future. I also want to thank the students of the scientific research camp. You share with me in life and give me reminders and help in learning. In the process of writing the paper, give me guidance in writing ideas and empirical analysis. In particular, I want to thank my family. It is because of your support that I can successfully complete this paper. Here I wish you good health and happiness. Finally, I would like to thank the original authors of all the documents I have learned from in this film paper, and thank you for giving me reference and help in the process of writing my paper.

\section{REFERENCES}

[1] Ping Lihua. Development of Visual Guidance System for Buttonhole Sewing Robot [D]. 2019.

[2] Gao Pan. Research on Delta Robot Buttonhole Sewing Technology [D]. Xi'an University of engineering, 2019.

[3] Wang Yongzhong, Wang Yanping, song Lingyun, etc. Current Situation and Development of Clothing Equipment Automation [J]. Electronic Technology, 2013, 26 (10).

[4] Wu Liubo, Li Xinrong, Du Jinli. Progress of Research on Trajectory Planning of Sewing Robot Based on Contour Extraction [J]. Journal of textile, 42 (4): 10.

[5] Feng Yingjie, Jiang Gaoming, Peng Jiajia. Artificial Intelligence Leads the Innovation and Development of the Textile Industry [J]. Modern Textile Technology, 29 (3): 7.

[6] Jiang Hongde. Personalized Customization in Clothing Driven by Data [J]. China informatization, 2017, 000 (003): 60-62. 